Ildeberto Santos-Ruiz

Tecnológico Nacional de México,

IT Tuxtla Gutiérrez,

TURIX-Dynamics Diagnosis and Control Group,

Carr. Panam. km 1080 S/N

Tuxtla Gutierrez 29050, Mexico;

Institut de Robòtica i Informàtica Industrial,

CSIC-UPC

Universitat Politècnica de Catalunya,

C/. Llorens I Artigas 4-6,

Barcelona 08028, Spain

e-mail: idelossantos@ittg.edu.mx

Francisco-Ronay LópezEstrada

TURIX-Dynamics Diagnosis and Control Group,

Tecnológico Nacional de México,

IT Tuxtla Gutiérrez,

Carr. Panam. km 1080 S/N,

Tuxtla Gutierrez 29050, Mexico

e-mail: frlopez@ittg.edu.mx

Vicenç Puig

Institut de Robòtica i Informàtica Industrial,

CSIC-UPC,

Universitat Politècnica de Catalunya,

C/. Llorens I Artigas 4-6,

Barcelona 08028, Spain

e-mail: vicenc.puig@upc.edu

Lizeth Torres

Instituto de Ingeniería,

Universidad Nacional Autónoma de México,

Ciudad de México 04510, Mexico

e-mail: ftorreso@iingen.unam.mx

\title{
Guillermo Valencia-Palomo
}

Tecnológico Nacional de México,

IT Hermosillo. Av. Tecnológico y Periférico

Poniente, $\mathrm{S} / \mathrm{N}$

Hermosillo 83170, Mexico

e-mail: gvalencia@hermosillo.tecnm.mx

\section{Samuel Gómez-Peñate}

TURIX-Dynamics Diagnosis and Control Group,

Tecnológico Nacional de México,

IT Tuxtla Gutiérrez,

Carr. Panam. km $1080 \mathrm{~S} / \mathrm{N}$

Tuxtla Gutierrez 29050, Mexico

e-mail: sgomez@ittg.edu.mx

\section{Introduction}

Pipelines are the major fluid transportation systems, and their proper management involves diagnosis, control, optimization, among other tasks [1,2]. These tasks are based on algorithms designed from physical laws that describe the behavior of the flows and pressure along the pipes, which have proven to work quite well. However, they have a significant disadvantage because it is necessary to update the model's parameters frequently. In particular, in pipes that have been in operation for a considerable

\footnotetext{
${ }^{1}$ Corresponding author.

Contributed by the Fluids Engineering Division of ASME for publication in the Journal OF Fluids Engineering. Manuscript received August 12, 2020; final manuscript received December 2, 2020; published online February 9, 2021. Assoc. Editor: Praveen Ramaprabhu.
}

time, two physical settings change drastically compared with the initial design: the roughness and the inner diameter. These changes are caused principally by the corrosion and wear of the internal walls, which vary due to the accumulation of mineral particles. The variation of these parameters affects the energy dissipation in a pipeline, and consequently, the physical model is no longer representative $[3,4]$. In pipelines, the energy dissipation caused by the friction is called head loss, which can be divided into major and minor losses. Major losses are associated with the loss of energy by roughness and viscosity, and minor losses are associated with elbows, flanges, or valves that change the flow direction causing dissipation of energy. The most common equation for calculating major losses is the Darcy-Weisbach (DW) equation [5], where the head loss is parameterized by the socalled Darcy-Weisbach friction factor, which in this paper will be 
named only as "friction factor." There are no sensors or transducers to directly measure the friction factor, so it must be calculated or estimated from other flow variables. The Blasius correlation equation [6] provides a practical way to determine the friction factor from the Reynolds number. However, this is only valid for smooth pipes because it does not consider the pipeline roughness [7]. When roughness is considered, the most accepted method to calculate the friction factor is the Colebrook-White (CW) equation, which unfortunately contains an implicit and transcendent function with no analytical solution in terms of elementary functions. In the literature, there are many explicit approximations to the $\mathrm{CW}$ equation, which computes the friction factor with different accuracy levels, e.g., the equations proposed by authors of Refs. [8-10]. The main drawback of these methods is that, as the $\mathrm{CW}$ equation, they require accurate roughness and inner diameters along the pipeline. Nevertheless, these parameters cannot be estimated easily [11].

In the literature, some works evaluate different methods to compute the friction factor from approximate solutions of the Colebrook-White equation, e.g., Refs. [12-16]. Nonetheless, the evaluations are usually focused only on the numerical analysis of the approximation errors, without considering experimental data of real pipelines and assuming known roughness coefficients. Also, these comparative analyses do not usually include friction calculation by the Lambert $W$-function $[17,18]$ in the comparison, possibly because this method is less known for its relatively recent publication. In Refs. [19] and [20], an analysis of the approximation error when calculating friction by Lambert $W$-function is presented. However, the study focuses on some low-order approximations of this function, detracting from its capabilities as an exact solution of the $\mathrm{CW}$ equation, especially considering that with the current high-performance computational systems (e.g., MATLAB) high order approximations can be obtained in a few nanoseconds. From this background, a new comparative analysis of the most used methods for estimating friction is needed, including the computation with the Lambert $W$-function, and assessing with measurements of a physical pipeline, not only from a theoretical point of view. This paper proposes a method for optimal estimation of the roughness coefficient and friction factor of pipelines. First, the depth analysis of several methods (including the computation with Lambert $W$-function) illustrates the main advantages and disadvantages of the $\mathrm{CW}$ approximations. Numerical evaluations are done by computing the percentage error between the results of the evaluated methods and those obtained by iterative solution of the $\mathrm{CW}$ equation, which is used as the standard reference. Experimental evaluation is carried out by considering experimental data of a laboratory pipeline. The percentage error for each method is calculated from the results that each one produces. The experimental friction obtained is computed from measurements of pressure at the ends of the pipeline. Second, a methodology to estimate the average roughness in a pipe by solving a nonlinear optimization problem, using least squares, is proposed. The estimated roughness is used to compute the friction by the different methods that are evaluated in this paper. The main motivation to systematize the friction computation procedure is that the Instituto Tecnológico de-Tuxtla Gutiérrez (ITTG) is currently working on the analysis and design of algorithms for leak diagnosis in pressurized pipelines, which depend on precise estimates of the friction. In previous work [4,21-23], the roughness coefficient used is either the one declared by the pipe manufacturer or it has been calculated from a single measurement of flow and pressures at the ends of the pipeline. However, in practice, it has been found that the roughness coefficient calculated this way is quite sensitive to changes in the operating point. Therefore, in this paper, a technique is proposed to estimate the roughness by nonlinear optimization from multiple measurements of pressure and flow in the pipeline.

This work is organized as follows: In Sec. 2, the theoretical basis of the head loss and friction is presented, including the DW equation and the CW equation. In Sec. 3, some known approaches to calculate the friction factor are presented; both exact and approximate methods are considered. In Sec. 4, a method for estimating roughness in a pipeline using nonlinear optimization is proposed. In Sec. 5, the numerical evaluation of the friction computation methods are presented; in this section, the result of the roughness estimation for a laboratory pipeline using the proposed method and the assessment of the approaches to computing the friction from the roughness estimated in the laboratory pipeline are also presented. Finally, in Sec. 6, some conclusions are presented, as well as the direction that the research that motivated the presentation of this paper will take.

\section{Theoretical Foundation}

A general assumption is that the head loss $\left(h_{f}\right)$ due to friction depends on the inner diameter $(D)$ of the pipe, the length $(L)$ in which the head loss is measured, the average flow speed $(V)$, the absolute roughness of the pipe wall $\left(k_{s}\right)$, the density $(\rho)$, and the viscosity of the fluid. By considering force balance and dimensional analysis, it is possible to determine the head loss ( $\mathrm{Pa})$ due to friction as $[24,25]$

$$
h_{f}=\frac{L}{D} \frac{\rho V^{2}}{2} F(\varepsilon, \mathrm{Re})
$$

where Re is the Reynolds number, which depends on viscosity and $\varepsilon=k_{s} / D$ is the so-called relative roughness coefficient (dimensionless). The expression $F(\varepsilon, \mathrm{Re})$ is the friction factor, which is represented by $f$ in this paper. In general, Eq. (1) is represented in the following simplified form called the DW equation $[5,24]$ :

$$
h_{f}=f \frac{L}{D} \frac{\rho V^{2}}{2}=f \frac{8 L \rho}{\pi^{2} D^{5}} Q^{2}
$$

where $Q$ is the volumetric flow rate in the pipeline. Equation (2) only predicts significant losses due to fluid friction on the pipe wall and due to the effects of fluid viscosity and does not include minor losses on inputs, elbows, and other fittings. Concerning different formulas for calculating friction losses, Eq. (2) has the advantage of being a dimensionally homogeneous equation, so that the friction factor $f$ is a dimensionless number consistent with any system of units [24]. Equation (1) is the most general equation for fluid resistance along the pipe. Therefore, any other formulation used to calculate friction losses is necessarily a particular case of Eq. (1).

If pressure sensors are available at the pipeline ends, then the head loss $h_{f}$ of Eq. (2) can be calculated by $h_{f}=H_{\text {in }}-H_{\text {out }}$, where $H_{\text {in }}$ and $H_{\text {out }}$ denote the upstream and the downstream pressure head measurements, respectively. If the flow rate of $Q$ is measured, then the friction factor can be computed by solving Eq. (2). The only requirement for computing the friction in this way is that the flow and pressures must be in a steady-state because other factors affect the energy dissipation in the transitory. In that case, it is necessary to know the function $F(\varepsilon, \operatorname{Re})$ that represents the friction factor $f$ in Eq. (1).

The search for a function $F(\varepsilon, \mathrm{Re})$ that allows calculating the friction factor as a function of roughness has generated different empirical equations. Thereby, von Kármán and Prandtl [26] found that when the flow is hydraulically smooth $(\varepsilon \rightarrow 0)$, the friction depends only on the Reynolds number and can be calculated using

$$
\frac{1}{\sqrt{f}}=2 \log _{10}(\operatorname{Re} \sqrt{f})-0.8
$$

whereas for the hydraulically rough flow, which corresponds to high turbulence, the following von Kármán's equation [27] can be used:

$$
\frac{1}{\sqrt{f}}=-2 \log _{10}(\varepsilon)+1.14
$$


According to Ref. [26], the flow is completely rough if $\varepsilon \operatorname{Re} \sqrt{f}>200$, and the hydraulically smooth flow condition corresponds to $\varepsilon \operatorname{Re} \sqrt{f} \leq 10$. For the transitional region between the smooth and rugged regimes, Eqs. (3) and (4) are not valid, because $f$ has a more complicated dependency of $\varepsilon$ and Re. Colebrook combined both Eqs. (3) and (4) into one [28,29] and also considers the friction in the transitional region. In the Colebrook approach, the simple sum of the right sides of these equations allows to cover both flow conditions, and smoothly interpolate between these extreme conditions. The resulting expression, known as the $\mathrm{CW}$ equation is given by

$$
\frac{1}{\sqrt{f}}=-2 \log _{10}\left(\frac{\varepsilon}{3.7}+\frac{2.51}{\operatorname{Re} \sqrt{f}}\right)
$$

In Ref. [18], it has been pointed out that, strictly, the way used by Colebrook to combine Eqs. (3) and (4) is mathematically incorrect, since $\log (x+y) \neq \log (x)+\log (y)$. However, Eq. (5) has been widely accepted for its empirical validity, since it has been experimentally verified. Figure 1 shows how the $\mathrm{CW}$ equation generalizes the equations of von Kármán and Prandtl, including the transitional region. In general, the $\mathrm{CW}$ equation is used in the domain defined by $(\varepsilon, \operatorname{Re}) \in\left\{4000 \leq \operatorname{Re} \leq 10^{8}\right.$ and $0 \leq \varepsilon \leq 0.05\}$.

Nevertheless, a drawback of Eq. (5) to compute $f$ is that its dependence on $\operatorname{Re}$ and $\varepsilon$ is implicit and nonlinear. Therefore, its solution requires iterative numerical methods (fixed point, bisection, among others), which reduces its applicability in real-time because the iterations require a considerable computational effort. One way to overcome this issue is through explicit approximations of $f$ obtained from Eq. (5). Among the most used approximations, there are those of Refs. [8-10]. In Sec. 3, these approximations are described, then in Sec. 5 an assessment of each of these approximations is presented, considering the times needed for its calculation and its error concerning the exact value determined by the implicit formula (5). The iterative solutions of the Colebrook-White equation are graphically represented in the so-called Moody chart [30], which allows to visually estimate the friction factor from the Reynolds number and the pipeline roughness. However, it is inaccurate and impractical when calculations must be automated within an algorithm. Nowadays, that graphical approach is only useful for educational purposes and a rough first estimation. Recently, analytical (closed-form) solutions of nonlinear equations similar to Eq. (5) have been found in terms of the Lambert $W$-function [18,31]. The main advantage of this approach is that it makes it possible to calculate the friction factor accurately by means of noniterative expressions. In Sec. 3, a formulation that explicitly determines $f$ based on this method is considered.

\section{Friction Factor Computation}

This section describes different approaches used to estimate the friction factor in the turbulent regime, from the Reynolds number and the pipeline roughness, which are assumed known.

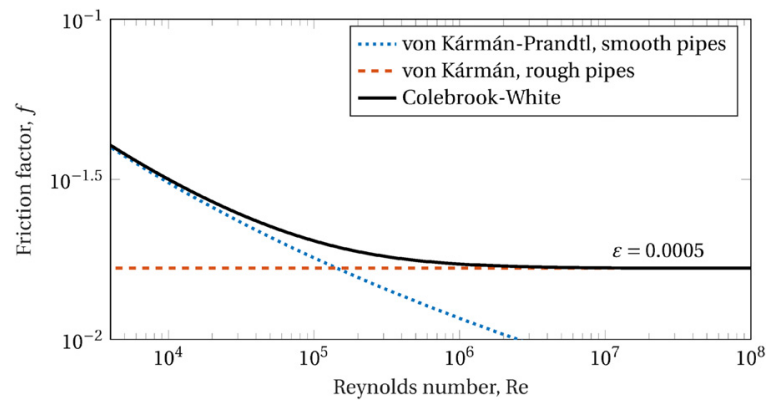

Fig. 1 Relationship between the Colebrook-White equation and the equations of von Kármán and Prandtl
3.1 Iterative Solution of the Colebrook-White Equation. The fast way to compute $f$ from the Colebrook-White equation is to use an iterative numerical method. For this, the nonlinear Eq. (5) is rewritten as

$$
\phi(f):=\frac{1}{\sqrt{f}}+2 \log _{10}\left(\frac{\varepsilon}{3.7}+\frac{2.51}{\operatorname{Re} \sqrt{f}}\right)=0
$$

Then, the problem is reduced to find the zero of $\phi(f)$ or, equivalently, the minimum of $\phi^{2}(f)$. A simple iterative approach to find the zero of $\phi(f)$ is through a fixed point iteration

$$
\begin{gathered}
f^{(0)}=1 \\
f^{(i+1)}=0.25 / \log _{10}\left(\varepsilon / 3.7+2.51 /\left(\operatorname{Re} \sqrt{f^{(i)}}\right)\right)^{2}
\end{gathered}
$$

Nevertheless, if the initial condition is not chosen properly, iterative procedures can converge toward "false solutions" due to the nonlinearity of Eq. (6), e.g., by considering open iterative methods as the Newton-Raphson [32]. For this reason, it is more convenient to use closed iterative methods that bound the solution on a delimited interval, for example, as in the bisection and linear interpolation methods. A convenient interval to search a solution of Eq. (6) could be $\left[0^{+}, 1\right]$. A strategy that in some cases shortens the convergence of open iterative methods is taking an initial estimation of $f^{(0)}$ the "false solution" of the CW equation by considering $\operatorname{Re} \rightarrow \infty$, which also corresponds to the value of $f$ provided by the von Kármán Eq. (4) for completely rough flow, such as

$$
f^{(0)}=0.25 / \log _{10}(\varepsilon / 3.7)^{2}
$$

As reported in Ref. [33], the initial approximation (9) has a maximum relative error of $80 \%$ over the application domain of the $\mathrm{CW}$ equation. Therefore, the number of iterations is considerably reduced with respect to the unitary initial estimation given in Eq. (8). Nevertheless, when there is an estimate or expected value of the friction in the operating conditions, such as $\hat{f}=0.02$ or similar (depending on the pipe material and the typical flow rate), this can be used as $f^{(0)}$. An implementation of the iterative fixedpoint solution of the $\mathrm{CW}$ equation using a spreadsheet is described in Ref. [34]. Regarding the iterative solutions of Eq. (6) based on the Newton-Raphson method, a $\mathrm{C}++$ implementation is described in Ref. [35].

3.2 Friction Estimation Using Explicit Approximations. Several formulas have been proposed in order to find an approximate value of $f$ replacing the $\mathrm{CW}$ equation by simplified versions where the dependence of $f$ on $\varepsilon$ and Re is already explicit. These simplifications allow direct computations of $f$ in a single step without requiring any iteration. For instance, the Swamee-Jain (10) and Haaland (11) approximations [8,9] are the most popular

$$
\begin{gathered}
f \approx 0.25 /\left(\log _{10}\left(\varepsilon / 3.7+5.74 / \operatorname{Re}^{0.9}\right)\right)^{2} \\
f \approx 1 /\left(-1.8 \log _{10}\left((\varepsilon / 3.7)^{1.11}+6.9 / \operatorname{Re}\right)\right)^{2}
\end{gathered}
$$

Typical errors of Eqs. (10) and (11) are less than 1\%, which is sufficient for many applications. However, in such cases, when greater accuracy is required, these estimations can be used as an initial approximation in iterative solutions of the $\mathrm{CW}$ equation to accelerate the convergence of iterative methods. In Ref. [36], a multistep approximation of $f$ was proposed by considering the Steffensen method. By considering this approach, the formula of Ref. [10] is considered to estimate $f$ more accurately than Eqs. (10) and (11). The following sequence of operations describes this method:

$$
A=-2 \log _{10}(\epsilon / 3.7+12 / \mathrm{Re})
$$




$$
\begin{gathered}
B=-2 \log _{10}(\epsilon / 3.7+2.51 A / \mathrm{Re}) \\
C=-2 \log _{10}(\epsilon / 3.7+2.51 B / \mathrm{Re}) \\
f \approx 1 /\left(A-(B-A)^{2} /(C-2 B+A)\right)^{2}
\end{gathered}
$$

A characteristic of this method is that it requires a fixed number of auxiliary values (three) before evaluating the final approximation. Consequently, the computational cost is also fixed. In addition, due to the fact that fractional powers are not involved, the computational time is quite close to the approximations given in Eqs. (10) and (11). Based on the Serghides equations (12a)-(12d), Cojbašić and Brkić [37] proposed an improved version by adjusting the model coefficients using genetic algorithm techniques. As a result, the coefficient 12 was changed to 12.585 , and 3.7 was changed to 3.71. Thus, the maximal relative error in estimated $f$ decreased 53.26 times compared to coefficients proposed by Serghides. The optimization of different explicit approximations of the $\mathrm{CW}$ equation using genetic algorithms is described in detail in Ref. [38].

Other approximate noniterative solutions of the CW equation have been proposed by the authors of Refs. [20,39-41], and [42], among others. An exhaustive list of noniterative approximations is not presented here, Eqs. (10), (11), and (12d) are cited for later comparison because they are often used. The common factor in noniterative methods is that they only approximate the value of $f$ with different degrees of accuracy. Section 3.3 describes a technique for calculating $f$ exactly.

3.3 Friction Computation Using the Lambert $W$-Function. In Ref. [43], it is proved that it is possible to express an explicit solution of Eq. (5) by the Lambert $W$-function. To the best of our knowledge, this is the only explicit solution of the $\mathrm{CW}$ equation as reported in Ref. [33]. The Lambert $W$-function is defined as the root of

$$
W(z) \mathrm{e}^{W(z)}=z
$$

for every complex number $z$ [44]. The mapping $z \mapsto W$ is a noninjective function (multivalued, except in $z=0$ ), which is frequently used to solve equations that contain exponentials or logarithms, as is the case with the $\mathrm{CW}$ equation. If real numbers are considered only, with constraints $\{z \geq-1 / \mathrm{e}, W(z) \geq-1\}$, then one-valued function $W_{0}(z)$ is obtained that it is also called as the "principal branch" of $W(z)$. In order to compute the friction factor from the $\mathrm{CW}$ equation, only the part $W_{0}^{+}$located in the first quadrant of the plane is considered (see Fig. 2).

The Lambert $W$-function can be formally expressed in an endless form, such as $[19,45]$

$$
\begin{array}{cc}
W(z)=\ln (z / \ln (z / \ln (z / \ln (\cdots)))), & \text { if }|W(z)|>1 \\
W(z)=z / \exp (z / \exp (z / \exp (\cdots))), & \text { if }|W(z)|<1
\end{array}
$$

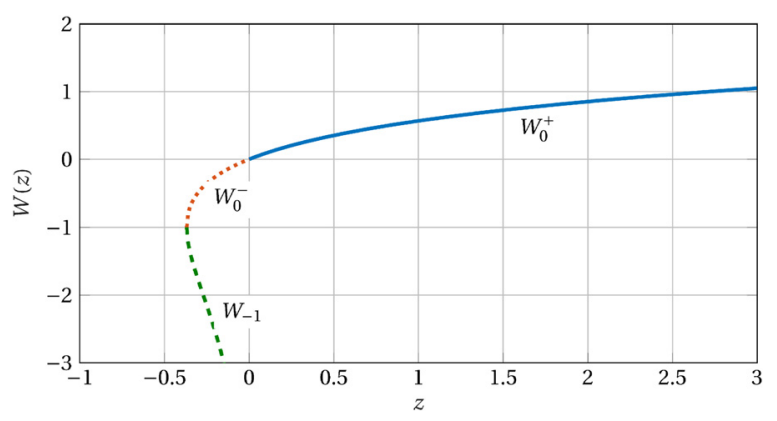

Fig. 2 Lambert $W$-function. The graph shows the neighborhood of the origin where $W(z)$ is divided into two branches. For larger $z$ values, $W_{0}(z)$ shows a logarithmic monotonous tendency.
However, from a practical point of view, methods based on truncated series are often used [44-47]. For instance, by considering the series expansion at $z=0$, the principal branch $W_{0}$ can be expressed by $[19,48]$

$$
\begin{aligned}
W_{0}(z) & =z-z^{2}+\frac{3}{2} z^{3}-\frac{8}{3} z^{4}+\frac{125}{24} z^{5}-\frac{54}{5} z^{6}+O\left(z^{7}\right) \\
& =\sum_{n=1}^{\infty} \frac{(-n)^{n-1}}{n !} z^{n}
\end{aligned}
$$

Although the series (16) is useful for small values of $z$, it is useless in the context of the $\mathrm{CW}$ equation because, as will be discussed later, the argument of $W_{0}(z)$ when calculating the friction factor is quite large $(z \gg 1)$. In this case, the following asymptotic behavior is most helpful

$$
W_{0}(z) \sim \ln (z)-\ln (\ln (z)), \quad \text { as } z \rightarrow \infty
$$

Approximation (17) gives good results when $z$ is quite large, but its accuracy decreases rapidly as $z$, so it is not suitable to use it to compute the friction factor over wide operating ranges. Therefore, to ensure accuracy in the calculated value of $W_{0}(z)$ throughout the range of interest, and implementation of the Lambert $W$-function based on Halley's method [49] is considered. Applying this method, the value $w=W_{0}(z)$ can be obtained by iterating through the following formula [50]:

$$
\begin{gathered}
f_{i}=w_{i} \exp \left(w_{i}\right)-z \\
w_{i+1}=w_{i}-\frac{f_{i}}{\left(w_{i}+1\right) \exp \left(w_{i}\right)-\frac{\left(w_{i}+2\right) f_{i}}{2 w_{i}+2}}
\end{gathered}
$$

Because Halley's method is cubically convergent, the accuracy in successive values of $w_{i}$ from Eq. (19) increases rapidly. In an exhaustive test, using Eq. (19) it was possible to calculate $W_{0}(z)$ in the whole interval $z \in(0, \infty)$ with a maximum of four iterations, starting from the coarse initial estimate $w_{0}=1$. Based on the performance of Halley's method reported in Ref. [50], Eqs. (18) and (19) with an improved initial approximation $w_{0}=$ $\ln (z)-\ln (\ln (z))$ from Eq. (17) was used to implement the Lambert $W$-function in this work.

Based on the Lambert $W$-function, in More [17] an explicit solution of the $\mathrm{CW}$ equation was proposed, which could predict the pressure drop in the flow of ideal gases through pipelines. Later, in Refs. [31] and [18] were reported the application of $W_{0}(z)$ in hydraulic problems involving the calculation of the friction factor by considering algebraic manipulations and some variable changes in Eq. (5) together with the $W_{0}$ function, a closed-form solution for $f$ was obtained, such that

$$
\begin{aligned}
f & =F(\varepsilon, \operatorname{Re}) \\
& =\frac{1}{\left((2 / \ln (10)) W_{0}\left((\ln (10) / 2 a) 10^{b / 2 a}\right)-b / a\right)^{2}}
\end{aligned}
$$

with $a=2.51 / \operatorname{Re}$ and $b=\varepsilon / 3.7$. The expression (20) is the only one explicit solution known for the $\mathrm{CW}$ equation.

Even though Eq. (20) provides a direct way to obtain the exact value of $f$, its use in diagnosis and control applications requires that the programming language used have native subroutines to evaluate the Lambert $W$-function numerically. In recent versions of MATLAB, Maple and $\mathrm{C}++$ compilers, these calculations can be programed directly. ${ }^{2}$ However, some drawbacks are computing $f$ when the product $\varepsilon \mathrm{Re}$ is large. This issue arises due to limitations

\footnotetext{
${ }^{2}$ To compute the Lambert $W$-function, some $\mathrm{C}++$ compilers may require the installation of the GNU Scientific Library.
} 
of the floating-point numerical system typically used in calculations. A strong limitation is that on IEEE-compliant computers the largest double-precision floating-point value [51] according to the IEEE 754 standard [52] is

$$
\text { DOUBLE_MAX }=1.797693134862316 \times 10^{308} .
$$

Due to this limitation, some combinations of Re and $\varepsilon$ prevent the numerical evaluation of the friction factor by Eq. (20), as already warned by Sonnad and Goudar [53], whereas the argument of $W_{0}$ in Eq. (20) should not exceed the maximum floatingpoint value (21). Nevertheless, it can be shown that to calculate $f$ by using the Lambert $W$-function in a double-precision IEEE machine, it must be satisfied that

$$
\ln (\mathrm{Re})+0.1239681863 \varepsilon \operatorname{Re}<710.5621104 .
$$

Since the constraint (22) is equivalent to the one published by Ref. [53], this condition will be cited later as "Sonnad-Goudar condition." The feasible region of Eq. (20) can be calculated as displayed in Fig. 3. Note that due to the fact that the feasible region (dashed line) is a logarithmic curve, logarithmic scales are used in the graph axes. In practice, Eq. (22) and Fig. 3 are useful because they can provide a simple method to determine the applicability of the Lambert $W$-function in explicit calculations of the friction factor. Some transformations of Eq. (20) that partially solve the problems in the computation of $f$ due to limitations in the floating point system (shaded region in Fig. 3) are described in Ref. [54]. To avoid the limitations in the direct evaluation of Eq. (20), strategies have also been proposed to compute the Lambert $W$-function based on truncated series that achieve good accuracy when approximating the power series with three or more terms, as proposed in Ref. [47].

Recently, a transformation of the CW equation in terms of $\varpi$ function (a shifted logarithmic version of $W$-function) has been proposed by Clamond [31]

$$
\frac{1}{\sqrt{f}}=\frac{2}{\ell} \varpi\left(\frac{\ell \epsilon \operatorname{Re}}{18.574} \mid \ln \left(\frac{\ell \operatorname{Re}}{5.02}\right)\right)
$$

where $\mid$ is a shift operator such that $\varpi(a \mid b)=W(\exp (a+b)-a)$, and $\ell=\ln (10)$. Using the Clamond's transformation (23) of the $\mathrm{CW}$ equation, the convergence problems when applying the Lambert $W$-function are solved. In Ref. [31], a fourth-order iteration is proposed to compute the friction factor from Eq. (23) in only two iterations. As will be shown later (in Sec. 5), the friction computation using Eq. (23) is fast and absolutely convergent throughout the region of interest for the turbulent flow.

All the methods discussed in this section, approximate and exact, can be computed by the MATLAB routines given in Ref. [55], including the one based on the Lambert $W$-function (Halley's method) and Clamond's transformation.

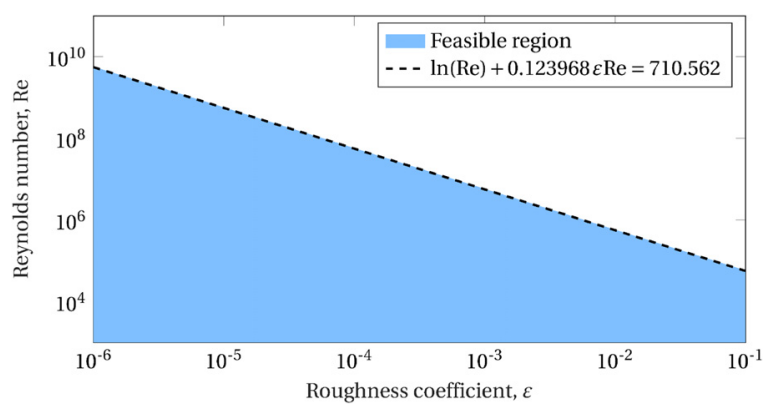

Fig. 3 Feasible region where it is possible to compute $f$ directly using the Lambert $W$-function

\section{Estimation of the Roughness Coefficient}

In the preceding section, it has been assumed that the computation of $f$ is carried out from known values of $\operatorname{Re}$ and $\varepsilon$. Nonetheless, even though the Reynolds number can be calculated in terms of the measured flow rate, the value of $\varepsilon$ cannot be calculated straightforwardly because $\varepsilon$ indicates how rough or smooth the internal walls of the pipeline are, which depends on the materials and the techniques used to manufacture the pipe. Furthermore, this parameter is only determined when the pipeline is installed, but under continuous operation, the walls of the pipe can be eroded or coated with particles carried by the flow. Therefore, a periodic "recalibration" of $\varepsilon$ is convenient [56]. This work proposes a method to estimate the relative roughness coefficient of $\varepsilon$. Initially, a set of pressure and flow measurements $\left(H_{\mathrm{in}}, H_{\mathrm{out}}, Q\right)$ for different operating points in a steady-state is obtained. In the experimental pipeline, the operating point is changed, employing a frequency inverter that regulates the power of a centrifugal pump that drives the flow through the pipeline. Then, for each operating point, the pressure loss is determined by $h_{f}=$ $H_{\text {in }}-H_{\text {out }}$ together with the flow $Q$, which are substituted in the Darcy-Weisbach Eq. (2) to compute the friction factor

$$
f=\frac{\pi^{2} D^{5} h_{f}}{8 L \rho Q^{2}}
$$

The value of $\mathrm{Re}$ is also calculated for each operating point, as

$$
\operatorname{Re}=\frac{D Q}{A \nu}
$$

where $\nu$ is the kinematic viscosity of the fluid, and $A$ is the crosssectional area of the pipe. Afterward, with the values of $f$ and $\operatorname{Re}$ obtained from the measurements in $N$ different operating points, the data array shown in Table 1 is constructed. Finally, the dataset $\left(\operatorname{Re}_{k}, f_{k}\right)$ is fitted to the explicit formula $f=F(\varepsilon, \mathrm{Re})$ of Eq. (20) solving the following nonlinear optimization problem:

$$
\hat{\varepsilon}=\operatorname{argmin}_{\varepsilon} \sum_{k=1}^{N}\left(F\left(\varepsilon, \operatorname{Re}_{k}\right)-f_{k}\right)^{2}
$$

Nevertheless, instead of $F(\varepsilon, \mathrm{Re})$ in Eq. (20), it is also possible to define the objective function of Eq. (26) by considering the explicit approximations of $f$ defined in Eqs. (10), (11), and (12d). Trust-region methods [57,58] and Levenberg-Marquardt algorithms $[59,60]$ are applicable to solve the least squares problem (26). Despite that theoretically it is possible to use a single pair $(\mathrm{Re}, f)$ to estimate $\varepsilon$ by solving Eq. (26), this results in biased estimates. Therefore, samples from several operating points $(k>1)$ must be used. Also, the pressure and flow measurements used to compute $\operatorname{Re}_{k}$ and $f_{k}$ at each operating point must be obtained by averaging the sensor signals over a time interval (about 100 samples), in order to minimize the effect of the measurement noise.

During the iterative process to minimize the cost function

$$
\mathcal{C}(\varepsilon):=\sum_{k=1}^{N}\left(F\left(\varepsilon, \operatorname{Re}_{k}\right)-f_{k}\right)^{2}
$$

Table 1 Data required to estimate $\varepsilon$

\begin{tabular}{ccccc}
\hline \hline$H_{\text {in }}{ }^{\mathrm{a}}$ & $H_{\text {out }}{ }^{\mathrm{a}}$ & $Q^{\mathrm{a}}$ & $\operatorname{Re}^{\mathrm{b}}$ & $f^{\mathrm{c}}$ \\
\hline$H_{\text {in1 }}$ & $H_{\text {out1 }}$ & $Q_{1}$ & $\operatorname{Re}_{1}$ & $f_{1}$ \\
$H_{\text {in2 }}$ & $H_{\text {out2 }}$ & $Q_{2}$ & $\operatorname{Re}_{2}$ & $f_{2}$ \\
$\vdots$ & $\vdots$ & $\vdots$ & $\vdots$ & $\vdots$ \\
$H_{\text {inN }}$ & $H_{\text {out } N}$ & $Q_{N}$ & $\operatorname{Re}_{N}$ & $f_{N}$ \\
\hline \hline
\end{tabular}

${ }^{\mathrm{a}}$ Measured data.

${ }^{\mathrm{b}}$ Calculated from Eq. (25).

${ }^{\mathrm{c} C a l c u l a t e d}$ from Eq. (2). 
it is quantified a first-order optimality measure (FOOM) to determine how close each new estimate $\epsilon^{(i)}$ is to the optimal value $\hat{\varepsilon}$. The infinity norm of the gradient of $\mathcal{C}(\varepsilon)$ is used as FOOM

$$
\mathrm{FOOM}=\max _{k}\left|(\nabla \mathcal{C}(\varepsilon))_{k}\right|=\|\nabla \mathcal{C}(\varepsilon)\|_{\infty}
$$

As an optimality measure, the FOOM is based on the idea that for a smooth function to reach a minimum, its gradient must be zero. Therefore, it is expected that the FOOM will continually decrease during the iterative optimization process until reaching a maximum permitted value (tolerance) $\mathrm{FOOM}_{\text {tol }}$ previously established. Other tolerance values that are also considered in the criterion to complete the iterative process are $\varepsilon_{\text {tol }}$ and $\mathcal{C}_{\text {tol }}$, which represent the maximum changes allowed in $\varepsilon$ and $\mathcal{C}(\varepsilon)$ during the iterations. Then, the iterations are completed if any of the following conditions are achieved:

$$
\begin{gathered}
\left|\varepsilon^{(i)}-\varepsilon^{(i-1)}\right|<\varepsilon_{\text {tol }} \\
\left|\mathcal{C}\left(\varepsilon^{(i)}\right)-\mathcal{C}\left(\varepsilon^{(i-1)}\right)\right|<\mathcal{C}_{\text {tol }}
\end{gathered}
$$

In the ROUGHNESS MATLAB-routine given in Ref. [55], the tolerances have the following predefined values: $\mathrm{FOOM}_{\text {tol }}=1 \times 10^{-12}, \varepsilon_{\text {tol }}=1 \times 10^{-9}$, and $\mathcal{C}_{\text {tol }}=1 \times 10^{-9}$. The Levenberg-Marquardt algorithm is used to solve Eq. (26) in these routines.

\section{Results and Discussion}

In this section, a numerical and experimental evaluation of the friction calculation methods described in section "Friction factor computation" is presented. The roughness estimation for a laboratory pipeline is also presented. This estimate is used for the experimental assessment of the methods described in Sec. 3.

5.1 Numerical Evaluation. To determine the accuracy of the explicit approximations (10), (11), and (12d), these were evaluated on a regular grid of $1000 \times 1000$ points in the region defined by

$$
4 \times 10^{3} \leq \operatorname{Re} \leq 1 \times 10^{8}, \quad 1 \times 10^{-6} \leq \varepsilon \leq 5 \times 10^{-2}
$$

The grid defined by Eq. (31) corresponds to the region mentioned in Sec. 2 where the CW equation is usually used. However, the limit case $\varepsilon=0$ was not used in the region discretization, because only rough pipes were considered. The results were contrasted with the exact values of $f$ obtained by the iterative solution of the Colebrook-White equation. The percentage errors of the different approximations are shown graphically in Figs. 4-6. In general, the explicit approximations of $f$ show a tendency to present some error for small roughness coefficients and Reynolds numbers close to laminar flow. As it can be seen, comparing the three approximations, the Serghides has a less percentage error

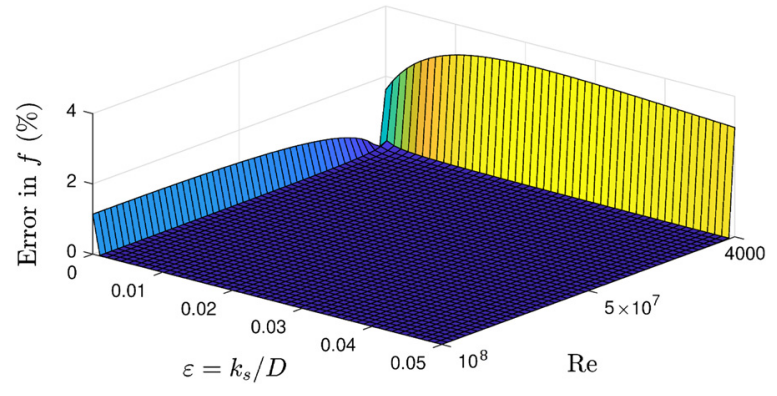

Fig. 4 Error in the Swamee-Jain approximation

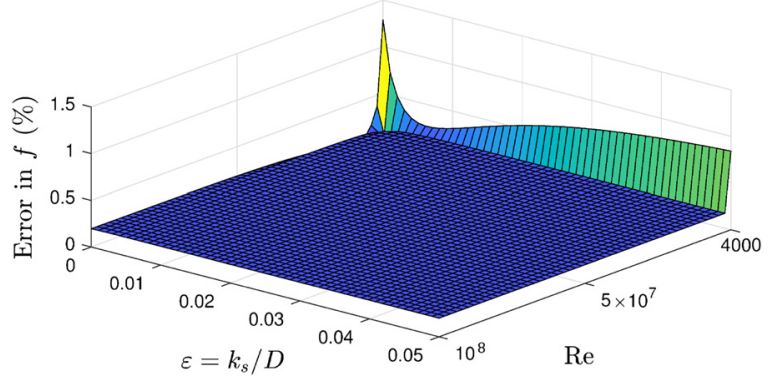

Fig. 5 Error in the Haaland approximation

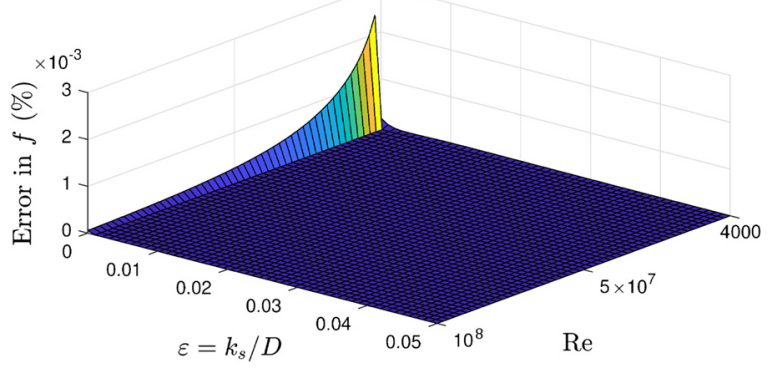

Fig. 6 Error in the Serghides approximation

than the Swamee-Jain and the Haaland approximations, with three orders of magnitude of difference.

A summary of the results is presented in Table 2, where in addition to the approximation error for each method, the computational time required to complete one million evaluations of $f$ over the grid described above is also shown. Active calculation times were measured using the profiler tool of MATLAB. The Swamee-Jain and Haaland approximations have a simpler structure than the Serghides approximation, so they should be computed in less time. However, it is important to note that the Swamee-Jain and Haaland equations contain fractional powers, unlike the Serghides equations which include only integer powers, which means that the time needed to compute the three approximations is quite close since the computational cost to calculate fractional powers is high because it is implemented using the relationship

$$
x^{a}=\exp (a \ln (x)), \quad x, a \in \mathbb{R}, \quad a \notin \mathbb{Z}
$$

Considering Eq. (32), to compute the approximations of Swamee-Jain, Haaland, and Serghides, it is necessary to evaluate three transcendental functions (and other basic operations) in each case, hence the closeness in their computation time.

The results in Figs. 4-6, as well as in Table 2, show that the Serghides method presents the best global performance since its computation time is comparable to the Swamee-Jain and the Haaland methods, but with a considerably smaller error. On the other hand, Eq. (20) based on the Lambert $W$-function give results as accurate as the iterative solution, but in MATLAB presents some convergence problems for highly turbulent flows for simultaneously using large roughness coefficients. Although this condition (large Re and large $\varepsilon$ ) rarely occurs in practice, it is important to consider this weakness for MATLAB implementations. The convergence region where it was possible to compute the friction factor using the MATLAB floating-point arithmetic is shown in Fig. 7. In the tests, the convergence region coincided punctually with the feasible region delimited by the Sonnad-Goudar condition (22). The estimation of $f$ given by Eq. (20) evaluates to zero in the nonconvergence region marked by a red cross $(\times)$. A logarithmic grid of $20 \times 20$ points was used to discretize the space $(\varepsilon, \operatorname{Re})$. As 
Table 2 Performance of different methods to estimate the friction factor

\begin{tabular}{|c|c|c|c|c|}
\hline Method & Average error $(\%)$ & Maximum error (\%) & Time $^{\mathrm{a}}(\mathrm{ms})$ & Convergence \\
\hline Iterative $^{\mathrm{b}}$ & 0 & 0 & 707 & Entire region (31) \\
\hline Swamee-Jain & 0.0148 & 3.3583 & 94 & Entire region (31) \\
\hline Haaland & 0.1997 & 1.4203 & 100 & Entire region (31) \\
\hline Serghides & $5.26 \times 10^{-7}$ & 0.0031 & 87 & Entire region (31) \\
\hline Lambert $W$-function $^{\text {c }}$ & $8.05 \times 10^{-13} \sim 0$ & $7.36 \times 10^{-12} \sim 0$ & 131 & Only in region (22) \\
\hline Clamond & $5.96 \times 10^{-14} \sim 0$ & $2.85 \times 10^{-13} \sim 0$ & 73 & Entire region (31) \\
\hline
\end{tabular}

${ }^{\mathrm{a}}$ Using an Intel Core i5 computer with $8 \mathrm{~GB}$ of RAM.

${ }^{\mathrm{b}}$ Using fixed-point method. Initial value according to Eq. (8) and tolerance $\varepsilon=2.2204 \times 10^{-16}$.

${ }^{\mathrm{c}}$ Halley's implementation, using 64-bit fixed precision arithmetic.

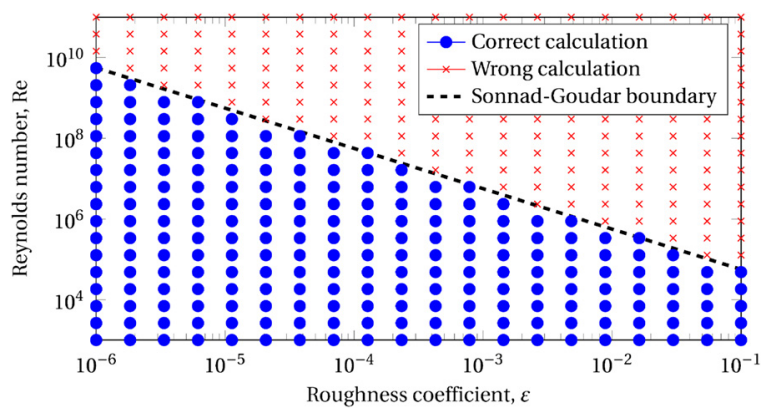

Fig. 7 Region of convergence when calculating the friction factor by Lambert $W$-function, using MATLAB with 64-bit fixed precision arithmetic

shown in Fig. 7, the main advantage of the friction computation using the Lambert $W$-function is due to the fact that converges exactly in the region where other methods present larger errors (for small Re and small $\varepsilon$ ).

Friction calculations using the Lambert $W$-function were also performed in Maple without convergence problems throughout the test region. Since Maple implements variable precision arithmetic, the convergence problems when applying Eq. (20) in MAT$\mathrm{LAB}$ are attributed to the 64-bit fixed precision arithmetic that MATLAB uses. In fact, by installing the Symbolic Math Toolbox in MATLAB, which implements variable precision arithmetic, it was possible to compute precisely the friction values throughout the test region using Eq. (20). On the other hand, it was found that Clamond's implementation also effectively solves convergence problems by calculating friction using the Lambert $W$-function even with fixed precision arithmetic.

5.2 Roughness Estimation From Experimental Data. As described before, an instrumented experimental pipeline was considered to test the proposed method as shown in Fig. 8. Because this pipeline is not straight, but has a serpentine shape, minor losses in elbows have been measured experimentally at each operating point. In the calculations, these losses have been subtracted

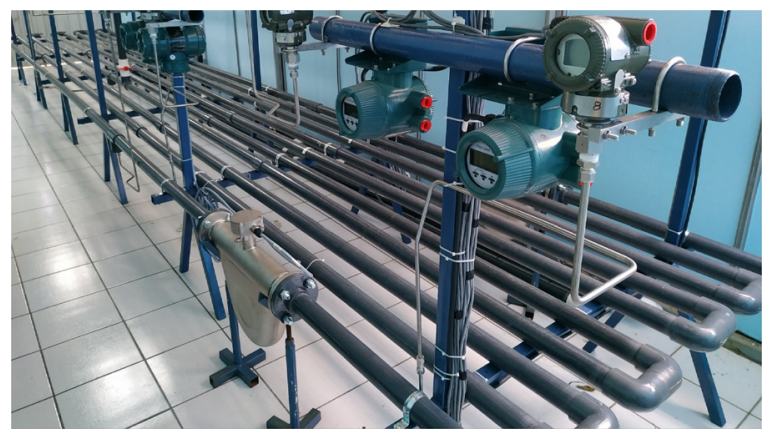

Fig. 8 Experimental pipeline in ITTG (Mexico)
Table 3 Experimental data used to estimate the relative roughness in the laboratory pipeline

\begin{tabular}{lcccc}
\hline \hline$H_{\text {in }}(\mathrm{m})$ & $H_{\text {out }}(\mathrm{m})$ & $Q\left(\mathrm{~m}^{3} / \mathrm{s}\right)$ & $\operatorname{Re}$ & $f$ \\
\hline 2.1826 & 1.2299 & 0.0014567 & 47,525 & 0.022786 \\
3.7503 & 1.5707 & 0.0022904 & 74,725 & 0.021086 \\
5.7087 & 1.9998 & 0.0030494 & 99,490 & 0.020241 \\
8.0448 & 2.5267 & 0.0037705 & 123,013 & 0.019698 \\
\hline \hline
\end{tabular}

from the input pressure $H_{\text {in }}$, so that only the major losses are used to calculate the roughness. In the experimental setup, the relative roughness coefficient was determined according to the procedure described in Sec. 4 from the measurements at four different operating points obtained with a centrifugal pump of $5 \mathrm{hp}$ working at frequencies of $25 \mathrm{~Hz}, 35 \mathrm{~Hz}, 45 \mathrm{~Hz}$, and $55 \mathrm{~Hz}$. Table 3 shows the data obtained from the pressure and flow sensors, as well as the values derived that were used for the estimation of $\varepsilon$ by considering the least squares method. Other parameters of the experimental pipeline used in the calculations are $L=64.48 \mathrm{~m}$, $D=0.0486 \mathrm{~m}, \nu=8.03 \times 10^{-7} \mathrm{~m}^{2} / \mathrm{s}$, and $g=9.79 \mathrm{~m} / \mathrm{s}^{2}$. The kinematic viscosity of water, $\nu$, was estimated from temperature measurements using the MATLAB code given in Ref. [61].

The solution to the optimization problem (26) using the Levenberg-Marquardt algorithm computes a relative roughness coefficient $\hat{\varepsilon}=4.8093 \times 10^{-4}$. Table 4 shows partial results of the iterative process, where the third to fifth columns include values related to the stop criterion defined by the tolerances considered on Eqs. (28)-(30). In Fig. 9, the exact solution of the Colebrook-White equation is contrasted for the estimated value of $\hat{\varepsilon}$ against the experimental values of $f$ obtained directly from the Darcy-Weisbach equation; the graphs cover only the interval $45,000 \leq \operatorname{Re} \leq 125,000$ corresponding to the operating region in the pipeline. The root-mean-square error in the estimation of $f$ for the four operating points was $\mathrm{RMSE}_{f}=4.4669 \times 10^{-5}$. Figure 9 also includes the curve of $f$ for the computed values of $\hat{\varepsilon}$ calculated from a single operating point. In this case, the error increased to $\operatorname{RMSE}_{f}=1.1164 \times 10^{-4}$ (almost three times greater), so there is evidence of the need to estimate $\varepsilon$ considering several operating points of pipeline, to avoid biased estimates. Table 5 numerically shows the decrease in RMSE when the number of pressure and flow measurements used to estimate $\hat{\varepsilon}$ increases from one to four. Nevertheless, in case of errors due to biased measurements, the use of multiple operating points does not necessarily reduce the error; this requires a recalibration of the measuring instruments.

5.3 Assessment of Estimated Friction Using Experimental Data. Figure 10 shows a different estimation of $f$ that incorporate the estimated roughness $\hat{\varepsilon}$ for the operating region of the laboratory pipeline, and also compares them against the experimental value of $f$ computed directly from the Darcy-Weisbach equation. The graphs locally confirm that the Serghides approximation coincides almost precisely with the exact solution of the Colebrook-White equation and with the experimental values of $f$, 
Table 4 Iterations to compute $\hat{\varepsilon}$ using Levenberg-Marquardt optimization algorithm

\begin{tabular}{lccc}
\hline \hline Iteration, $i$ & $\varepsilon^{(i)}$ & $\mathcal{C}\left(\varepsilon^{(i)}\right)$ & FOOM $^{(i)}$ \\
\hline 0 & $2.22045 \times 10^{-16 a}$ & $3.09689 \times 10^{-10}$ & $1.48 \times 10^{-6}$ \\
1 & 0.000086643 & $1.26440 \times 10^{-10}$ & $7.12 \times 10^{-7}$ \\
2 & 0.000228718 & $1.82144 \times 10^{-11}$ & $1.53 \times 10^{-7}$ \\
3 & 0.000338907 & $1.65797 \times 10^{-12}$ & $2.40 \times 10^{-8}$ \\
4 & 0.000406107 & $1.24114 \times 10^{-13}$ & $3.30 \times 10^{-9}$ \\
5 & 0.000443330 & $8.74448 \times 10^{-15}$ & $4.29 \times 10^{-10}$ \\
6 & 0.000463627 & $6.73076 \times 10^{-16}$ & $5.40 \times 10^{-11}$ \\
7 & 0.000476059 & $8.83817 \times 10^{-17}$ & $5.47 \times 10^{-12}$ \\
8 & 0.000480145 & $6.43052 \times 10^{-17}$ & $7.52 \times 10^{-13}$ \\
9 & 0.000480890 & $6.37056 \times 10^{-17}$ & $3.72230 \times 10^{-4}$ \\
10 & 0.000480931 & $6.37033 \times 10^{-17}$ & $2.02973 \times 10^{-5}$ \\
11 & 0.000480933 & $6.37032 \times 10^{-17}$ & $1.24324 \times 10^{-5}$ \\
12 & 0.000480934 & $6.37032 \times 10^{-17}$ & $4.08537 \times 10^{-6}$ \\
\hline \hline
\end{tabular}

aMachine epsilon, used to avoid "division by zero" error.

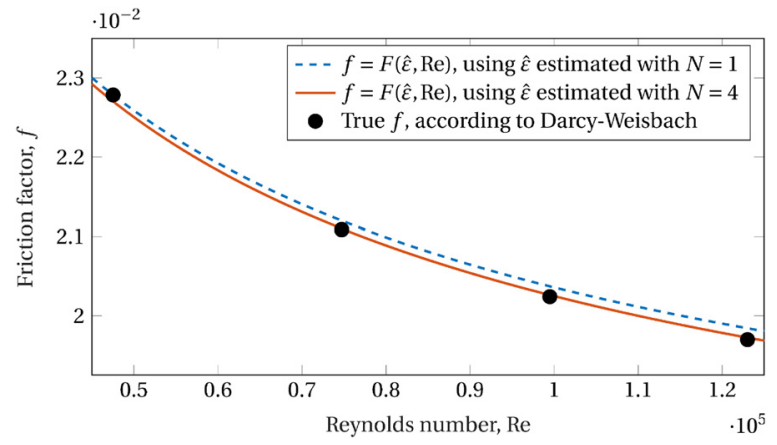

Fig. 9 Friction estimation by Colebrook-White equation using $\hat{\varepsilon}$ obtained from experimental data

Table 5 Estimated roughness using different number of operating points

\begin{tabular}{lcc}
\hline \hline$N$ & $\hat{\varepsilon}$ & $\mathrm{RMSE}_{f}$ \\
\hline 1 & $5.0763 \times 10^{-4}$ & $1.1164 \times 10^{-4}$ \\
2 & $4.8977 \times 10^{-4}$ & $5.6119 \times 10^{-5}$ \\
3 & $4.8378 \times 10^{-4}$ & $4.5988 \times 10^{-5}$ \\
4 & $4.8093 \times 10^{-4}$ & $4.4669 \times 10^{-5}$ \\
\hline \hline
\end{tabular}

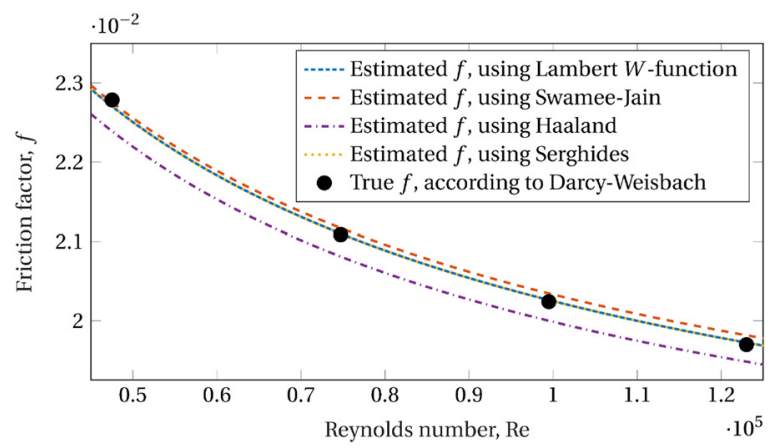

Fig. 10 Different estimates of $f$ in the operating region of the laboratory pipeline. The estimate with Lambert $W$-function and the Serghides approximation are almost equal, in the operating range of the prototype pipeline, so it is difficult to visualize both.
Table 6 Performance of different methods to compute $f$ using $\hat{\varepsilon}$ obtained from experimental data

\begin{tabular}{lc}
\hline \hline Method & RMSE $_{f}$ \\
\hline Iterative & $4.4669 \times 10^{-5}$ \\
Swamee-Jain & $9.0406 \times 10^{-5}$ \\
Haaland & $2.8996 \times 10^{-4}$ \\
Serghides & $4.4713 \times 10^{-5}$ \\
Lambert $W$-function & $4.4669 \times 10^{-5}$ \\
\hline \hline
\end{tabular}

thus outperforming the approximations of Swamee-Jain and Haaland that are frequently used in pipeline monitoring and leakage diagnosis algorithms. In fact, within the operating region of the experimental pipeline, the accuracy of the Serghides approximation is comparable to that obtained with the Lambert $W$-function. As a result, it can be concluded that Lambert $W$-function has better performance, except for the convergence problems described previously, and also because it is a "nonelementary" function, it could not be available in some programming languages.

Table 6 shows the improvement when using the Lambert $W$ function instead of the other methods, for the four operation points sampled in the laboratory pipeline. It is emphasized that the RMSE when using the Lambert $W$-function to calculate the friction is equal to the RMSE obtained by the iterative method, which is considered as the most accurate. Nevertheless, the Lambert $W$ function can be implemented online under the limitations described before.

The $\mathrm{RMSE}_{f}$ reported in Table 6 only quantifies the propagation of the estimation error $\varepsilon-\hat{\varepsilon}$ when applying the different calculation methods of $f$ without considering the measurement error in pressure and flow variables. The total error in $f$ may increase due to measurement uncertainty, which in the instruments of the pilot pipeline is between $0.01 \%$ and $0.04 \%$, so these values impose an upper limit on the accuracy of the computed value of $f$. Regarding the influence of measurement uncertainty on the error when computing $f$, the sensitivity analysis for the operating range of the pilot pipeline produces

$$
\begin{gathered}
\frac{\Delta f}{f}=0.092 \frac{\Delta \varepsilon}{\varepsilon} \\
\frac{\Delta f}{f}=-0.156 \frac{\Delta \operatorname{Re}}{\operatorname{Re}}
\end{gathered}
$$

where $(\Delta \operatorname{Re}) / \operatorname{Re}$ can be taken equal to the flow rate measurement error, $(\Delta Q) / Q$, considering the relationship (25). These results show that the friction calculation is considerably sensitive to flow measurement errors. 


\section{Conclusions}

The problem of determining the friction factor $f$ for the turbulent flow was addressed considering three approaches: the iterative solution of the Colebrook-White equation, the use of explicit approximations of $f$ (Swamee-Jain, Haaland, and Serghides) and the exact calculation of $f$ using the Lambert $W$-function. In the analysis, the latter was the best option, because it requires less processing time and it is an exact solution of the $\mathrm{CW}$ equation. However, its implementation is limited by the calculation of $f$ for programming languages where there is no subroutine to evaluate $W_{0}$, e.g., in some embedded systems, the Serghides approximation is also a good option. In the analysis of the dependence of $f$ on the roughness coefficient $\varepsilon$ in the experimental pipeline, it was found that the actual value of this one can differ considerably from the value reported by the manufacturer (when it exists, in the best of cases) or of the one assumed according to the tables or manuals of hydraulics. Therefore, the roughness coefficient must be estimated experimentally. The procedure that was proposed to adjust $\varepsilon$ in the experimental pipeline produced good results, given that the value of $f$ derived from it coincides with the value obtained from the measurements using the Darcy-Weisbach equation. However, since the pipeline roughness can vary with time, the periodic application of this procedure is recommended as a recalibration action. As future work, it has been considered to experiment with different pipe materials and perform an analysis of the effect that the different approximations of the friction factor have on algorithms for leak diagnosis. Also, research will be done to consider their influence of the friction on the accuracy of the fault diagnosis methods when estimating the flow rate and the leak position (analysis of the error propagation), as well as their computational demands in terms of the total processing time to implement the diagnostic algorithms in real-time. It is also contemplated to extend the roughness estimation methodology for pipeline networks, which represents a challenging problem because few pressure and flow measurements are available in a hydraulic system.

\section{Funding Data}

- National Council for Science and Technology (CONACYT) of Mexico (under the program Atención a Problemas Nacionales) (Grant No. PN-2016/3595).

\section{References}

[1] C., Verde, and L. Torres, eds., 2017, Modeling and Monitoring of Pipelines and Networks: Advanced Tools for Automatic Monitoring and Supervision of Pipelines (Applied Condition Monitoring), Vol. 7, Springer International Publishing, Berlin.

[2] Puig, V., Ocampo-Martinez, C., Pérez, R., Cembrano, G., Quevedo, J., and Escobet, T., 2017, Real-Time Monitoring and Operational Control of DrinkingWater Systems, Springer, Berlin.

[3] Torres, L., and Verde, C., 2018, "Nonlinear Estimation of a Power Law for the Friction in a Pipeline," Second Conference on Modelling, Identification and Control of Nonlinear Systems (MICNON 2018), Guadalajara, Mexico, June, IFAC, pp. 67-72.

[4] Santos-Ruiz, I., Bermúdez, J., López-Estrada, F., Puig, V., Torres, L., and Delgado-Aguiñaga, J., 2018, "Online Leak Diagnosis in Pipelines Using an Ekf-Based and Steady-State Mixed Approach," Control Eng. Pract., 81, pp. $55-64$.

[5] Weisbach, J., 1845, "Lehrbuch der Ingenieur-und Maschinen," Mechanik, 1, p. 434.

[6] Trinh, K. T., 2010, "On the Blasius Correlation for Friction Factors," arXiv preprint arXiv:1007.2466.

[7] Von Bernuth, R., 1990, "Simple and Accurate Friction Loss Equation for Plastic Pipe,” J. Irrig. Drain. Eng., 116(2), pp. 294-298.

[8] Swamee, P. K., and Jain, A. K., 1976, "Explicit Equations for Pipe-Flow Problems," J. Hydraulics Div., 102(5), pp. 657-664.

[9] Haaland, S. E., 1983, "Simple and Explicit Formulas for the Friction Factor in Turbulent Pipe Flow," ASME J. Fluids Eng., 105(1), pp. 89-90.

[10] Serghides, T. K., 1984, "Estimate Friction Factor Accurately," Chem. Eng., 91(5), pp. 63-64.

[11] Santos-Ruiz, I., López-Estrada, F.-R., Puig, V., and Valencia-Palomo, G., 2020, "Simultaneous Optimal Estimation of Roughness and Minor Loss Coefficients in a Pipeline," Math. Comput. Appl., 25(3), p. 56.

[12] Zigrang, D., and Sylvester, N., 1985, "A Review of Explicit Friction Factor Equations," ASME J. Energy Resour. Technol., 107(2), pp. 280-283.
[13] Y Ild Ir Im, G., 2009, “Computer-Based Analysis of Explicit Approximations to the Implicit Colebrook-White Equation in Turbulent Flow Friction Factor Calculation," Adv. Eng. Software, 40(11), pp. 1183-1190.

[14] Brkić, D., 2011, "Review of Explicit Approximations to the Colebrook Relation for Flow Friction," J. Pet. Sci. Eng., 77(1), pp. 34-48.

[15] Genić, S., Arandelović, I., Kolendić, P., Jarić, M., Budimir, N., and Genić, V., 2011, "A Review of Explicit Approximations of Colebrook's Equation," FME Trans., 39(2), pp. 67-71.

[16] Zeghadnia, L., Robert, J. L., and Achour, B., 2019, "Explicit Solutions for Turbulent Flow Friction Factor: A Review, Assessment and Approaches Classification," Ain Shams Eng. J., 10(1), pp. 243-252.

[17] More, A. A., 2006, "Analytical Solutions for the Colebrook and White Equation and for Pressure Drop in Ideal Gas Flow in Pipes," Chem. Eng. Sci., 61(16), pp. $5515-5519$.

[18] Brkić, D., 2012, "Lambert W Function in Hydraulic Problems," Math. Balkanica, 26(3), pp. 285-292.

[19] Brkić, D., 2011, "W Solutions of the CW Equation for Flow Friction," Appl. Math. Lett., 24(8), pp. 1379-1383.

[20] Brkić, D., and Praks, P., 2018, "Accurate and Efficient Explicit Approximations of the Colebrook Flow Friction Equation Based on the Wright $\omega$-Function," Mathematics, 7(1), p. 34

[21] Bermúdez, J.-R., López-Estrada, F.-R., Besançon, G., Valencia-Palomo, G., Torres, L., and Hernández, H.-R., 2018, "Modeling and Simulation of a Hydraulic Network for Leak Diagnosis," Math. Comput. Appl., 23(4), p. 70 .

[22] Bermúdez, J. R., Santos-Ruiz, I., López-Estrada, F. R., Torres, L., and Puig, V., 2017, "Diseño y Modelado Dinámico de Una Planta Piloto Para Detección de Fugas Hidráulicas (Design and Dynamic Modeling of a Pilot Plant for Hydraulic Leak Detection)," Congreso Nacional de Control Automático CNCA 2017, Vol. 1, Asociación Mexicana de Control Automático, pp. 2-7.

[23] Pérez-Pérez, E. J., López-Estrada, F. R., Valencia-Palomo, G., Torres, L., Puig, V., and Mina-Antonio, J. D., 2021, "Leak Diagnosis in Pipelines Using a Combined Artificial Neural Network Approach," Control Eng. Pract., 107, p. 104677.

[24] Brown, G. O., 2002, The History of the Darcy-Weisbach Equation for Pipe Flow Resistance, ASCE, Washington, DC, pp. 34-43.

[25] Saldarriaga, J., 2016, Hidráulica de Tuberías, 3rd ed., Alfaomega, Mexico City, Mexico.

[26] Rouse, H., 1943, "Evaluation of Boundary Roughness," Proceedings Second Hydraulics Conference, Bulletin No. 27.

[27] Von Kármán, T., 1930, "Mechanische Änlichkeit und Turbulenz," Nachrichten Von Der Gesellschaft Der Wissenschaften zu Göttingen, MathematischPhysikalische Klasse, Math.-Phys. Kl., Göttingen, Germany, pp. 58-76.

[28] Colebrook, C. F., White, C. M., and Taylor, G. I., 1937, "Experiments With Fluid Friction in Roughened Pipes," Proc. R. Soc. London. Ser., A, 161(906), pp. 367-381

[29] Colebrook, C. F., 1939, "Turbulent Flow in Pipes, With Particular Reference to the Transition Region Between the Smooth and Rough Pipe Laws,” J. Inst. Civ. Eng., 11(4), pp. 133-156.

[30] Moody, L. F., 1944, "Friction Factors for Pipe Flow," Trans. ASME, 66, pp. 671-684.

[31] Clamond, D., 2009, "Efficient Resolution of the Colebrook Equation," Ind. Eng. Chem. Res., 48(7), pp. 3665-3671.

[32] Dahlquist, G., and Bjorck, A., 2008, Numerical Methods in Scientific Computing, Vol. 103, SIAM, Philadelphia, PA.

[33] Praks, P., and Brkić, D., 2018, "Advanced Iterative Procedures for Solving the Implicit Colebrook Equation for Fluid Flow Friction,” Adv. Civ. Eng., 2018, pp. $1-18$.

[34] Brkić, D., 2017, "Solution of the Implicit Colebrook Equation for Flow Friction Using Excel," Spreadsheets Educ., 10(2), pp. 1-12.

[35] Olivares, A., Guerra, R., Alfaro, M., Notte-Cuello, E., and Puentes, L., 2019 "Experimental Evaluation of Correlations Used to Calculate Friction Factor for Turbulent Flow in Cylindrical Pipes,” Rev. Int. Métodos Numér. Cálc. Diseño Ing., 35(1), p. 15.

[36] Jain, P., 2007, "Steffensen Type Methods for Solving Non-Linear Equations," Appl. Math. Comput., 194(2), pp. 527-533.

[37] Cojbašić, Ž., and Brkić, D., 2013, "Very Accurate Explicit Approximations for Calculation of the Colebrook Friction Factor," Int. J. Mech. Sci., 67, pp $10-13$.

[38] Brkić, D., and Cojbašić, Ž., 2017, "Evolutionary Optimization of Colebrook's Turbulent Flow Friction Approximations," Fluids, 2(2), p. 15.

[39] Buzzelli, D., 2008, "Calculating Friction in One Step," Mach. Des., 80(12), pp. $54-55$.

[40] Romeo, E., Royo, C., and Monzón, A., 2002, "Improved Explicit Equations for Estimation of the Friction Factor in Rough and Smooth Pipes," Chem. Eng. J., 86(3), pp. 369-374

[41] Praks, P., and Brkic, D., 2020, "Review of New Flow Friction Equations: Constructing Colebrook Explicit Correlations Accurately," arXiv preprint arXiv:2005.07021.

[42] Zigrang, D., and Sylvester, N., 1982, "Explicit Approximations to the Solution of Colebrook's Friction Factor Equation," AIChE J., 28(3), pp. 514-515.

[43] Keady, G., 1998, "Colebrook-White Formula for Pipe Flows," J. Hydraulic Eng., 124(1), pp. 96-97.

[44] Corless, R. M., Gonnet, G. H., Hare, D. E., Jeffrey, D. J., and Knuth, D. E., 1996, "On the Lambert W Function," Adv. Comput. Math., 5(1), pp. 329-359. 
[45] Corless, R. M., Jeffrey, D. J., and Knuth, D. E., 1997, "A Sequence of Series for the Lambert W Function," Proceedings of the International Symposium on Symbolic and Algebraic Computation (ISSAC), Vol. 97, Kihei, Maui, HI, pp. 197-204.

[46] Rollmann, P., and Spindler, K., 2015, "Explicit Representation of the Implicit Colebrook-White Equation," Case Stud. Therm. Eng., 5 pp. 41-47.

[47] Biberg, D., 2017, "Fast and Accurate Approximations for the Colebrook Equation,” ASME J. Fluids Eng., 139(3), p. 031401.

[48] Winitzki, S., 2003, "Uniform Approximations for Transcendental Functions," Third International Conference on Computational Science and Its Applications (ICCSA 2003), V. Kumar, M. L. Gavrilova, C. J. K. Tan, and P. L’Ecuyer, eds., Springer, Berlin, pp. 780-789.

[49] Gander, W., 1985, "On Halley's Iteration Method,” Am. Math. Mon., 92(2), pp. $131-134$.

[50] Veberič, D., 2012, "Lambert W Function for Applications in Physics," Comput Phys. Commun., 183(12), pp. 2622-2628.

[51] Press, W. H., Teukolsky, S. A., Vetterling, W. T., and Flannery, B. P., 2007, Numerical Recipes: The Art of Scientific Computing, 3rd ed., Cambridge University Press, Cambridge, UK.

[52] Zuras, D., Cowlishaw, M., Aiken, A., Applegate, M., Bailey, D., Bass, S. Bhandarkar, D., Bhat, M., Bindel, D., and Boldo, S., 2008, "IEEE Standard for Floating-Point Arithmetic,” IEEE, New York, Standard No. 754-2008.
[53] Sonnad, J. R., and Goudar, C. T., 2004, "Constraints for Using Lambert W Function-Based Explicit Colebrook-White Equation," J. Hydraulic Eng., 130(9), pp. 929-931.

[54] Brkić, D., 2012, "Comparison of the Lambert W-Function Based Solutions to the Colebrook Equation," Eng. Comput., 29(6), pp. 617-630.

[55] Santos-Ruiz, I., López-Estrada, F. R., and Puig, V., 2018, "Friction and Roughness," Zenodo.

[56] Echávez, G., 1997, "Increase in Losses Coefficient With Age for Small Diameter Pipes," J. Hydraulic Eng., 123(2), pp. 157-159.

[57] Powell, M. J., 1968, "A Fortran Subroutine for Solving Systems of Nonlinear Algebraic Equations," Atomic Energy Research Establishment, Harwell, UK Report No. AERE-R-5947.

[58] Coleman, T. F., and Li, Y., 1996, "An Interior Trust Region Approach for Nonlinear Minimization Subject to Bounds," SIAM J. Optim., 6(2), pp. 418-445.

[59] Marquardt, D. W., 1963, "An Algorithm for Least-Squares Estimation of Nonlinear Parameters,” J. Soc. Ind. Appl. Math., 11(2), pp. 431-441.

[60] Moré, J. J., 1978, "The Levenberg-Marquardt Algorithm: Implementation and Theory," Numerical Analysis, Springer, Berlin, pp. 105-116.

[61] Santos-Ruiz, I., López-Estrada, F. R., and Puig, V., 2019, "Estimation of Some Physical Properties of Water as a Function of Temperature," Zenodo. 